\title{
Orthodoxie et communisme dans les Balkans : réflexions sur le cas bulgare
}

Galia Valtchinova

\section{(2) OpenEdition}

12 Journals

Édition électronique

URL : http://journals.openedition.org/assr/2781

DOI : 10.4000/assr.2781

ISSN : $1777-5825$

Éditeur

Éditions de l'EHESS

\section{Édition imprimée}

Date de publication : 1 juillet 2002

Pagination : 79-97

ISBN : 2-222-96721-X

ISSN : 0335-5985

\section{Référence électronique}

Galia Valtchinova, "Orthodoxie et communisme dans les Balkans : réflexions sur le cas bulgare », Archives de sciences sociales des religions [En ligne], 119 | juillet - septembre 2002, mis en ligne le 05 décembre 2005, consulté le 19 avril 2019. URL : http://journals.openedition.org/assr/2781 ; DOI : $10.4000 /$ assr. 2781 


\section{ORTHODOXIE ET COMMUNISME DANS LES BALKANS : RÉFLEXIONS SUR LE CAS BULGARE*}

Au lendemain de la chute des régimes communistes en Europe du Sud-Est, l'Occident découvrait un monde où le religieux semblait retrouver une redoutable emprise sur les esprits. Depuis, cette région des Balkans ne cesse de présenter l'image d'une religion abusée à la fois par l'identitaire et le politique et dont les conflits à caractère religieux d'ex-Yougoslavie ne sont pas l'aspect le moins déconcertant. Les appartenances religieuses y sont affichées avec conviction, vécues avec ferveur et les contestations politiques s'y expriment à travers les déchirements confessionnels (1). Ce qui incite à considérer la religion dans les Balkans, et surtout dans le pays de confession orthodoxe qui fut le protagoniste de ces guerres, la Serbie, comme une sorte de bannière brandie par les fauteurs de conflits ethniques et politiques, sans autonomie sociale.

Sortir du paradigme identitaire de la religion c'est souvent renouer avec un autre réflexe explicatif, celui du renouveau religieux postcommuniste. Qu'il s'agisse de la Bulgarie ou de l'Albanie, c'est cette vision d'une religiosité entièrement étouffée par le régime totalitaire qui est souvent privilégiée. Les exemples empruntés à l'Europe centrale ont pourtant montré les limites, pour la sociologie du religieux, de la thèse de la "parenthèse communiste", thèse selon laquelle toute expression religieuse aurait été congelée jusqu'au "grand dégel" consécutif à la chute du mur de Berlin (2). Cette critique reste peu entendue - et étendue - des sociétés de tradition orthodoxe. La vision d'une tabula rasa imposée à la vie religieuse par le régime précédent reste implicite à la plupart des recherches sur le reli-

* Cet article résulte d'un travail sur le projet « Women and Religion in Balkan Christian Orthodox Context: the Communist Parenthesis? » financé par RSS grant n 994/2000, Open Society Fund-Prague.

(1) Les interprétations de cette nature sont abondantes : cf. SELls (1996, p. 13, passim) pour une caractérisation 'religieuse' de la guerre de Bosnie; RAMET (1996 [1992]) avec littérature.

(2) Comme le montre Patrick Michel (1994, pp. 11-13 [cf. la critique pp. 47-78]), cette vision est largement tributaire à la formule de l'«arrêt» de l'Histoire dans les pays de l'Europe centrale sous les régimes prosoviétiques, et leur «rentrée dans l'Histoire» (selon l'expression rendue célèbre par Havel) en 1989. 
gieux à l'époque postcommuniste. Cette vision met l'accent sur la répression de la religiosité chrétienne et sur les conséquences qui en découlent pour la société, et pour l'Église orthodoxe en particulier (3).

À côté de ces deux approches du religieux dans les sociétés orthodoxes qui ont vécu sous l'une ou l'autre des variantes du communisme, mutuellement exclusives à plus d'un égard, il faut en mentionner une troisième, inspirée de l'exemple du pays orthodoxe par excellence : la Grèce. État démocratique qui n'a pas connu de régime communiste, la Grèce constitue un terrain qui se prête à une approche beaucoup plus nuancée et ouverte aux problématiques du religieux des sociétés modernes. C'est dans ce «modèle grec» qu'on retrouve, à côté de la question du nationalisme inhérent à l'Église orthodoxe et à son alliance avec l'État, celle (qui ne se pose que rarement pour les pays ex-communistes) de la relation complexe entre la doctrine et les pratiques de l'orthodoxie d'un côté et la modernité, la sécularisation - de l'autre (4). Mettre côte à côte les différentes façons de traiter de la religion dans des sociétés qui, comme la Grèce, la Russie postsoviétique et la Serbie «saisie par le paroxysme nationaliste» (5), se réclament, chacune, d'une orthodoxie exemplaire, est une belle illustration des possibilités (mais aussi des limites) de chacune de ces approches.

Le choix de présenter le cas bulgare vient du fait qu'en ses différentes facettes, il offre de la matière à chacun des trois paradigmes explicatifs qui y prévalent à divers moments et avec un succès variable. En effet, au cœur des Balkans, la Bulgarie se revendique d'une tradition orthodoxe fort ancienne (antérieure à celles de Russie et de Serbie) directement rattachée à Byzance. À l'époque ottomane (fin $\mathrm{XIV}^{\mathrm{e}}$-XIX ${ }^{\mathrm{e}}$ siècles), l'orthodoxie bulgare est refondue à l'intérieur du Rum-millet (désignation ottomane des chrétiens orthodoxes assimilés aux Romaioi, ou Grecs byzantins), pour resurgir au milieu du XIX ${ }^{\mathrm{e}}$ siècle. Ces développements justifient l'approche identitaire de la religion orthodoxe à l'œuvre dans l'historiographie bulgare, ainsi que l'attention portée à l'implication de l'Église orthodoxe bulgare dans les mouvements nationaux. Ces aspects passent au second plan dès qu'il s'agit de l'époque communiste : parlant d'après 1944, on traite de la religion en Bulgarie en suivant le paradigme soviétique. En effet, seul pays des anciens satellites de l'URSS qui n'a jamais laissé se manifester un mouvement contestataire, la Bulgarie passe pour l'exemple d'un communisme docile et “orthodoxe" vis-à-vis de Moscou (6). La

(3) La vision dominante est celle d'une "mise à mort de la religion", orchestrée et conduite essentiellement dans les années trente et relancée au début des années soixante, et de l'anéantissement de la vie religieuse qui passe par la corruption des structures ecclésiastiques (voir par exemple MEYENDORFF, 1995, pp. 99-114). Les recherches les plus récentes semblent remettre en question la vision monolithique du "vide religieux" sous le régime soviétique, lui préférant des formules plus nuancées : cf. RoussELET (1997), ou "la religion à l'épreuve du communisme" dans BEAUVisage (1998, pp. 19-35).

(4) On peut découvrir la problématique très riche et variée de la recherche sur la religion et religieux contemporain en Grèce en mettant côte à côte les travaux de sociologues comme KoKosaLAKIS (1987a, 1987b, 1996) et MAKRIDES (1991) à ceux des anthropologues travaillant sur les terrains grecs Dubisch (1995); Herzfeld (1997b); Seremetakis (1991), Hart (1992). Sur la Russie, cf. Beauvisage (1998).

(5) Cf. les développements parallèles de Kokosalakis, Filatov et Vrcan dans Cipriani, ed. (1994), suivis du commentaire de VARGA (1994).

(6) CF. Lory (1996, pp. 62-64, 80-82); Crampton (1997, pp. 199-200); Fotev (1999); cf. CreED (1998, pp. 14-15). 
conformité avec le modèle soviétique est censée s'appliquer à toutes les sphères de la vie, y compris à la sphère du religieux. Serait-on face au mariage idéal entre deux orthodoxies, l'une qui relèverait de la docilité dans le domaine politique, l'autre d'une tradition partagée quant à la confession (7)? Promue par le discours politique, véhiculée par les médias, cette vision est largement partagée au sein de la société bulgare. La plupart des sciences sociales domestiques y souscrivent sans réserve (8). Or les études ethnographiques sur les réalités de l'époque socialiste (9), tout comme certains éléments du boom religieux d'après 1989 suggèrent que la vie religieuse entre le milieu des années 1940 et 1989 n'est pas réductible à la persécution, à la coercition ni au "vide religieux" (10). Il semble qu'ici comme dans d'autres domaines de la vie sociale, l'arrêt de l'histoire n'a pas eu lieu. Je me propose de voir, étude ethnographique à l'appui, ce qui s'est passé au cours de la période socialiste (11), et quelles étaient les facettes d'une vie religieuse qui continuait son cours sous les apparences de l'athéisme obligé.

Pour répondre à ces questions, j'interroge d'un côté le lien entre modernité et orthodoxie. De l'autre, je tente de démêler les subtilités du rapport entre religion et politique dans la société balkanique et communiste de la seconde moitié du $\mathrm{XX}^{\mathrm{e}}$ siècle. L'exposé est construit en deux sections afin d'articuler le passage du plan général à un tableau particulier. C'est ce dernier qui devrait permettre d'apprécier la portée des arrangements locaux entre régime communiste et religion chrétienne orthodoxe.

\section{Religion, modernité et politique : le contexte balkano-orthodoxe}

Il relève de l'axiome sociologique wébérien que la modernisation d'une société, ou sa modernité, s'exprime sur le plan religieux en une variété de phéno-

(7) Dans la même logique, on peut lier le "slavisme" à l" "orientalisme" supposé inhérent aux cultures slaves orthodoxes et que certaines spéculations attribuent à leur "inaptitude» fondamentale à la démocratie. C'est à ce type d'amalgame entre "traits culturels" tenus pour constants d'une part, et réalités sociales du moment de l'autre, que procèdent les promoteurs de thèses déterministes du "totalitarisme à l'est" versus "la démocratie à l'ouest". Pour une critique de cet essentialisme cf. Todorova (1997).

(8) Pour une vue d'ensemble sur la situation actuelle cf. Fotev (1999).

(9) Cf. Creed (1998); Petrov (1998); Valtchinova (1998).

(10) Il s'agit de la prolifération des activités des soi-disants 'extra-sensoriels', thérapeutes relevant à la fois du spécialiste religieux et du pratiquant de médecine alternative du genre New Age healing. On trouvera un état des lieux de cette question dans un numéro spécial de la revue Folklore Bulgare, 1995, $\mathrm{n}^{\mathrm{O}}$ 3. Une tendance analogue est observée en Russie postcommuniste, cf. White \& MCAlister (1997).

(11) À noter que les qualifications de [régime] 'communiste' ou 'totalitaire', et respectivement de 'communisme' et 'totalitarisme', préférées par les philosophes, les historiens et certains sociologues, reflètent le point de vue extérieur. Se positionnant à l'intérieur des sociétés étudiées, les anthropologues préfèrent d'une façon générale les termes de [régime] 'socialiste' ou de 'socialisme' (cf. CREED, 1998, avec la littérature). Dans ce texte, tout en accordant la préférence à ce dernier terme, les deux sont employés de façon interchangeable. 
mènes qui forment le couple célèbre désenchantement-sécularisation (12). Ces deux notions caractérisent les mouvements principaux à l'œuvre dans l'évolution de la religion, laquelle, sous la pression d'un rationalisme croissant, se retire de l'arène politique et de la sphère sociale pour se réfugier dans la sphère domestique et privée, provoquant ainsi la baisse ou la perte de la religiosité sous ses formes conventionnelles perceptibles à l'extérieur, ainsi que la séparation des Églises et de l'État. Indéniablement, la «mise en quarantaine» de la religion dans les premières décennies des régimes communistes, les attaques idéologiques et "l'athéisation» forcée de la population constituent un aspect de la sécularisation; on a pourtant mis en garde sur l'emploi du terme dans un contexte communiste (13). Il est plus sûr d'aborder la problématique par la modernité. Comme dans la plupart des pays balkaniques, la modernisation profonde de la société bulgare s'effectue après la Deuxième Guerre mondiale (14). Le fait que la quasi-totalité de cette période coïncide avec l'époque socialiste, communiste ou totalitaire selon les vocabulaires en cours, change peu cette configuration de départ. La superposition de processus sociaux de nature très différente est pourtant à l'origine de l'indistinction entre ce qui, sur le plan religieux, relève de la modernisation et ce qui est imputable aux abus d'un régime politique. Essayons de faire la part de chacun d'eux.

La proximité qui existe de nos jours, dans les sociétés orthodoxes, entre le politique et le religieux d'un côté, entre la religion et le nationalisme de l'autre, est le fruit d'une longue tradition qui remonte à l'Empire byzantin. À l'époque ottomane, l'Église doit s'adapter au fonctionnement d'un Empire fondé sur le principe des communautés religieuses (millets). Ceci renforce sa double identité, religieuse et politique, et fait de l'Église orthodoxe un enjeu dans les luttes de construction nationale au XIX ${ }^{\mathrm{e}}$ et début du $\mathrm{XX}^{\mathrm{e}}$ siècles; ce qui confère une légitimité intrinsèque, pour ainsi dire, à sa dimension «nationalitaire» (15). Dans la lignée de l'exemple serbe (le seul qui précède l'époque des 'Réveils' nationaux), mais aussi grec (à partir de 1833), l'Église 'nationale' devient un outil à penser et en même temps à justifier la continuité politique entre les sociétés balkaniques pré-modernes et les États-nations qui en émergent au cours du XIX ${ }^{\mathrm{e}}$ siècle. À l'heure de l'éveil national bulgare, la lutte pour la création d'une Église orthodoxe autonome (l'Exarcat bulgare) favorise cette vision d'une correspondance terme à terme. Cette lutte (1826-1870) spécifique participe de la construction nationale; vue du côté grec pourtant, elle scelle l'image d'une Église 'schismatique', instrument premier d'un nationalisme effréné. Le schisme bulgare ouvre la voie à une prolifération d'Églises nationales représentatives des millets qui se multiplient au sein de l'État ottoman. De nos jours, tous les pays orthodoxes se caractérisent par la correspondance terme à terme entre États nationaux et Églises. La collaboration entre Eglise et État y reste justifiée par les besoins de "l'idée nationale" (16).

(12) Le vocabulaire conceptuel utilisé est emprunté en grande partie à une sociologie du religieux d'inspiration wébérienne tournée vers la «modernité tardive»; ses repères sont exposés, entre autres, dans Hervieu-LÉger (1993) (cf. Arch. 84.47), Davie (1996) et Davie, Hervieu-Léger (1996).

(13) Cf. Tomka (1991), avec la littérature.

(14) Faisant l'objet d'un âpre débat au sein de l'intelligentsia bulgare (résumé dans FotEv, 1999), c'est un point sur lequel historiens et socio-anthropologues occidentaux sont d'accord : cf. LORY (1996, pp. 101-107); CREed (1998).

(15) Cf. Makrides (1991) et Papasthatis (1999), pour les développements sur le plan juridique.

(16) Les exemples d'une telle collaboration en Grèce, dans HerzFeld (1997b, pp. 69-75); Stewart (1998). Pour la Roumanie, Gillet (1997, pp. 17-73). 
On observe que toutes les sociétés orthodoxes balkaniques tendent à établir un lien étroit entre confession et identité nationale. Même à l'heure actuelle le Grec se conçoit, se met en scène, et se fait penser par les autres comme un orthodoxe par essence (17). L'actualité des guerres dans l'ex-Yougoslavie a consacré la vision d'un alliage de fer entre christianisme orthodoxe et identité serbe. Dans une autre veine, l'observation du 'terrain yougoslave' confirme le fait que la confession [orthodoxe] reste, même de nos jours, une affaire de choix - mais aussi de contraintes - collectifs, plus qu'une question de conviction ou de choix individuel (18). Cette observation s'applique à toutes les sociétés orthodoxes de la région, sans distinction du régime politique sous lequel elles vivent ou ont vécu (19). Or cette assimilation entre confession et identité collective ('ethnique' ou nationale selon les différents vocabulaires) réduit considérablement les effets de la sécularisation qui va de pair avec la privatisation du croire et de la pratique religieuse (20). Dans ce sens, les sociétés balkano-orthodoxes sont à ranger plutôt sous le label "appartenir sans croire" dans l'équation sociologique dont le pôle opposé, le "croire sans appartenir", est censé correspondre davantage à la religiosité des sociétés occidentales (21).

Un aspect pragmatique de la complicité entre l'Église orthodoxe et l'État, c'est le statut de fonctionnaire dont bénéficient les prêtres, qui perçoivent des salaires fixes. Dans tous les États balkaniques formés au cours du XIX siècle où l'orthodoxie est la confession dominante, le fonctionnariat est la base de la gestion interne des Églises. En Grèce, par exemple, une fois l'indépendance acquise (1830), le prêtre devient un employé de l'État. Il est enrôlé dans les entreprises de centralisation politique et d'homogénéisation culturelle entre des sociétés locales, jusqu'alors très fragmentées, et le pouvoir central (22), ou encore entre la société hellénique et les minorités chrétiennes. Ce rôle social (et parfois politique) ambigu du prêtre orthodoxe est une des justifications possibles d'un anticléricalisme populaire de longue date qui reste néanmoins tout à fait compatible avec la forte religiosité des sociétés traditionnelles (23). Il offre un terreau favorable au régime antireligieux qui instrumentalise le sentiment anticlérical enraciné dans les mentali-

(17) Cf. Kokosalakis (1987a, 1987b, 1996); Makrides (1991), Dubsich (1995), Bazin (2000, pp. 182-183) et pour une élaboration de ce point HERZFELd (1997). Pour une conception pareille du Roumain, cf. GiLlet (1997, pp. 91-102).

(18) CF. LORY (1996, p. 121). Selon SELls (1996) ce serait une particularité qu'on observe essentiellement chez les orthodoxes (Serbes).

(19) La réaction de l'EOB vis-à-vis de la pénétration des nouveaux mouvements religieux - mais aussi des dénominations protestantes - est identique à celle de l'Église grecque et des croyants grecs. Comme l'observent J. Dubisch (1995, p. 59) et M. Herzfeld (1997a, pp. 76-78), l'argument contre le choix individuel dans ce sens est la 'pollution' ou la 'trahison' vis-à-vis de sa propre communauté 'ethnique'.

(20) Sur la privatisation du croire cf. LuCKMAnN (1967) et Beyer (1990).

(21) Pour la formule cf. DAvie (1996, pp. 129-156).

(22) La signification de cette mesure, à la fois modernisatrice (car elle vise à surmonter les particularismes et à véhiculer la politique d'un État national) et conservatrice, a récemment été démontrée à propos de la région de Mani, en Grèce méridionale - cf. Seremetakis (1991, pp. 160-161). En effet, si le bas clergé (les pappas [curés de village]) est conçu, en Grèce depuis la guerre de l'Indépendance, comme le gardien et le garant de l'identité grecque (cf. MAKRIDES, 1991, pp. 284-289), ce même clergé est également beaucoup plus dépendant des autorités séculières que celui des pays catholiques. Cette observation vaut pleinement pour le cas bulgare.

(23) Pour la Grèce, cf. en particulier Just (1988); MAKRIDES (1991, p. 285 avec littérature). 
tés populaires (24). L'anticléricalisme populaire est riche de formes de dérision vis-à-vis de la figure du spécialiste religieux (le pope) que l'athéisme ne tardera pas à s'approprier. Il prépare le terrain pour cultiver les arguments "scientifiques" en faveur de l'athéisme, arguments présentés comme des impératifs de la modernité anti-chrétienne et anti-ecclésiastique, au nom du rationalisme (25). Or, comme on le sait, 1" "idéologie négative" de l'anticléricalisme est un axiome de la modernité (26).

Dans la modernité religieuse, les expressions extérieures - donc les mieux observables - de la religiosité sont susceptibles d'être en décalage tant par rapport à la conviction intime que par rapport à la pratique (27). Elles se laissent difficilement repérer et articuler à l'aide de critères et d'indices formels. Ce constat se vérifie d'une façon générale pour la vie religieuse en Bulgarie socialiste, surtout après les années soixante. Vers cette époque, des stratégies pour dissimuler la conviction et la pratique religieuse (surtout quand celle-ci est sporadique) sont déjà mises en place. Elles restent donc plus difficiles à repérer et à articuler. En fait, croyance et pratique (les deux notions prises au sens instrumental) peuvent être dissimulées autant par peur de la répression, qu'à cause d'un discours dominant qui fait rimer athéisme, ou abandon de la pratique religieuse, avec progrès, science et/ou modernité. Comme le montre David Kertzer (28), ce discours se fait entendre et inhibe l'expression de la foi religieuse même dans une Italie où, en dépit du vote communiste, le catholicisme ne fut jamais entièrement battu dans les banlieues "rouges" des villes industrielles du Nord. On peut facilement imaginer que la valeur normative de ce discours est encore plus forte dans une société préoccupée par son image de 'culture orientale' aux 'mœurs arriérées', et dont les élites, aussi bien avant que pendant le communisme, tiennent à renvoyer vers l'Europe occidentale l'image d'une société 'civilisée', donc moderne (29).

Ce raisonnement est appuyé par les résultats d'une étude sociologique menée, à la fin des années soixante et au début des années soixante-dix, au sein de cinq sociétés européennes, parvenues à des degrés de modernisation différents, et représentatives des trois traditions à l'intérieur de la chrétienté : catholicisme, protestantisme et orthodoxie. La Bulgarie est le seul pays communiste, caractérisé comme un "pays de modernisation relativement faible mais de changement révolutionnaire récent”. C'est également le seul pays de tradition orthodoxe. L'étude, centrée sur la relation entre la famille, le rituel et la sécularisation, permet d'observer des évolutions de la vie religieuse difficiles à relever par des indices formels. Les auteurs aboutissent à la conclusion que le processus de sécularisation est observé partout mais qu'il ne se présente pas comme un déclin général du religieux. Partout, les

(24) Selon P. Sugar (1989), les régimes communistes dans les Balkans sont anticléricaux, non antireligieux.

(25) C'est une des idées directrices dans les deux ouvrages sociologiques bulgares des années soixante : Ochavkov, éd. (1968), et Draganov (1968).

(26) René REMOND (1983). Sur le rapport entre anticléricalisme et modernité, je m'appuie sur le numéro spécial de European Studies Review, vol. 13, nº 2 [1983].

(27) Pour un développement théorique de ce point cf. HerVIEU-LÉger (1993).

(28) KERTZER (1980, pp. 64-65); cf. les observations analogues sur le terrain bulgare des années soixante dans Ochavkov, éd. (1970, pp. 59-60).

(29) Sur le lien entre modernité et occidentalisme vs. traditionalisme 'orientalisant', cf. M. HERZFELD (1997a, passim) et M. Todorova (1997, pp. 3-20). 
pratiques et les croyances "superstitieuses" semblent plus persistantes que la fréquentation de l'église et l'observation du culte administré par l'Église. On note qu'au fur et à mesure que la sécularisation avance, la "superstition" supplante les formes agréées pour ainsi dire du surnaturel, ou s'y superpose (30).

La dernière remarque introduit une autre constante de la vie religieuse en pays orthodoxe, à savoir la vitalité de la religiosité informelle. En Grèce, on note une abondance de formes extra-canoniques (dites 'populaires') de dévotion, aptes à évoluer afin de s'adapter aux canons les plus stricts (31). L'enchevêtrement entre la religion officielle et la profusion de formes populaires sous lesquelles elle s'exprime et qu'elle intègre, constitue une ligne de partage supplémentaire de l'orthodoxie par rapport aux autres christianismes. À elle seule, cette particularité fournit un argument central dans l'explication sociologique de l'absence de sécularisation, ou plutôt de son "inachèvement", en Grèce (32). Cette caractéristique est renforcée par le décalage entre religiosité personnelle et manifestation extérieure de la foi ou du sentiment religieux. D’une façon générale, les sociétés orthodoxes se montrent relativement tolérantes à l'égard de la non-observation stricte d'un comportement religieux extériorisé. L'exemple classique est la fréquentation de l'église : mise à part la messe dominicale, elle n'est pas vue comme une condition sine qua non du «bon chrétien» (33). L'assiduité du croyant se laisse facilement assimiler à un ensemble de gestes rituels et d'actes routiniers, et ne suppose pas une connaissance profonde de la doctrine chrétienne orthodoxe. C'est au contraire le dispositif matériel (comme les icônes) qui semble important, et la perception, le travail des sens l'emporte sur la réflexion. On note également l'abondance de repères matériels de cette religiosité enracinée dans le vécu : localiser un culte, fixer la dimension symbolique d'un lieu par une référence chrétienne, fait partie d'une façon de vivre la religion au même titre que fréquenter l'église, baiser les icônes, faire le signe de la croix en entendant les cloches sonner (34).

On remarque une nette tendance à mettre cet aspect pragmatique, et plus généralement matériel, de la religion en rapport avec la place de la femme dans la gestion de la vie religieuse au quotidien (35). De la recherche anthropologique sur la Grèce, il ressort, en plus, une nette différentiation sexuelle en ce qui concerne les indices de religiosité eux-mêmes, surtout en milieu rural. D'un côté, l'on constate que "la présence du prêtre mise à part, l'église reste un espace exclusivement féminin" (36). De l'autre, on observe que la fréquentation masculine de l'église en Grèce moderne obéit surtout à des stratégies de valorisation sociale et d'accumulation de prestige. D’une façon générale, la fréquentation masculine de l'église reste de basse intensité et manque de régularité, se limitant aux jours fériés, sinon à quelques grandes fêtes chrétiennes. En revanche, il est admis - voire obligatoire - que

(30) Cf. Luschen, Stajkof, Stolte Heiskanen \& Ward (1972 en particulier pp. 522-525).

(31) Kokosalakis (1987a, 1987b, pp. 227-228). Ce côté est particulièrement mis en lumière dans les études socio-anthropologiques : cf. Dubisch (1991 et 1995); Hart (1992), Seremetakis (1991).

(32) Cf. Kokosalakis (1987a; 1987b, 1996).

(33) Le cas de la communauté Sarakatsane étudiée par CAMPBEll (1964) est un exemple classique dans ce sens; cf. également HART (1992, pp. 147-151).

(34) Ces aspects de la religiosité orthodoxe sont examinés et soulignés dans les travaux d'anthropologues : cf. Hirschon (1983), Seremetakis (1991), Hart (1992), Dubisch (1995).

(35) Cf. en particulier Hirschon (1983), SerEMETAKis (1991, pp. 159-169), HART (1992 passim).

(36) Dubisch (1991, p. 42); cf. Hirschon (1983); Hart (1992, pp. 155-159). 
les hommes politiques fassent étalage de leur pratique de bons orthodoxes, surtout lors de la liturgie pascale. Cette stratégie sera suivie dès les premiers signes de libéralisation en Bulgarie où l'élite de l'ancien PC sera aussi assidue que la nouvelle élite démocratique à faire des apparitions publiques dans les grandes églises et les monastères (37). Dans tous les cas pourtant, c'est de l'habileté à exécuter les gestes essentiels du croyant et en fonction de la maîtrise du contexte social et temporel où ces gestes se placent qu'on conclut, en pays orthodoxe, sur le statut social et l'altérité possible d'une personne extérieure (38).

\section{Faire [sur]vivre le religieux dans la société communiste?}

\section{L'Église : entre répression et collaboration}

Dans la chronologie courante du communisme en Bulgarie (1944-1989), il serait prudent de prendre en compte le caractère transitoire de la période de l'immédiat après-guerre. C'est donc vers 1948 qu'on assiste à l'enracinement du pouvoir communiste, année qui marque le début de la répression religieuse, après la promulgation de la Loi de séparation de l'Église et de l'État (1947). Des persécutions directes sont lancées à des intervalles plus ou moins réguliers dans la décennie qui suit, de façon à faire sentir la volonté et la détermination de la force politique, désormais seul maître de la scène politique, à mettre en œuvre ses visions radicales concernant la religion. Il semble que parmi les vagues successives d'arrestation et d'intimidations qui s'abattent sur le clergé et les croyants, celle de 1948-1949 est la plus puissante (39). Malgré leur violence, les répressions contre le clergé orthodoxe restent de courte durée et sont souvent déclenchées à titre préventif. Il n'en va pas de même avec les structures chrétiennes non-orthodoxes implantées dans le pays : elles sont mises hors la loi, après des procès montés qui traduisent devant le tribunal populaire les organisations protestantes de diverses obédiences (1949) et les "prêtres catholiques" (1952) (40). L’Église Orthodoxe Bulgare [EOB] ne s'oppose pas aux persécutions contre les autres institutions chré-

(37) Cf. BAzIN (2000, p. 182) pour la Grèce; Fotev (1999, pp. 132-133) pour la Bulgarie.

(38) Par exemple savoir quand, comment et dans quelle posture faire le signe de la croix, allumer un cierge et le placer à l'endroit approprié, baiser l'icône etc., font partie d'un savoir-faire qui permet de distinguer sans faille l'habitant local du outsider en Grèce rurale. Cette observation de G. DuBISCH (1991; 1995, pp. 49-75, 101-110) a été vérifiée par l'auteur de ces lignes lors de son travail de terrain en Grèce du Nord.

(39) Cf. Crampton (1997, pp. 193, 194). Kalkandzhieva (1997, pp. 276-286) distingue trois vagues de répressions et de persécutions contre le clergé orthodoxe : la première juste après l'instauration du régime de transition (automne 1944-1945), l'autre consécutive à la promulgation de la nouvelle Constitution et de la Loi des confessions (fin 1948-1949), la dernière coöncidant avec le pic du durcissement du régime autoritaire (1952-1953).

(40) Cf. Kalkandzhieva (op. cit., pp. 281-285). Pour une perspective plus large, cf. CRAmPtON (1997, pp. 193, 194, 199). 
tiennes, se faisant discrète pour tout ce qui ne porte pas directement atteinte à ses propres intérêts (41).

Après quelques années d'alternance entre persécutions ouvertes et périodes de frictions plus ou moins violentes, les relations entre l'Église bulgare et l'État communiste commencent à se normaliser. Déjà en 1948, tout en proclamant la séparation entre l'État et l'Église et la confiscation de la plus grande partie des biens de celle-ci, le nouveau régime affiche un modus vivendi de relative tolérance vis-à-vis de l'EOB. En 1953, le chef de l'Église bulgare recouvre la dignité de Patriarche un regalium datant de l'époque médiévale - au terme d'un long processus de négociations avec le Patriarche œcuménique de Constantinople menées grâce à la médiation de Moscou (42). Cette reconnaissance met fin à la longue crise institutionnelle déclenchée par la création de l'Exarchat (1870) et la suppression de son siège à Istanbul/Constantinople (1913). C'est un événement marquant pour le prestige international de l'Église orthodoxe bulgare. Habilement, il est mis à profit par les nouvelles autorités politiques pour accumuler du capital symbolique pour leur propre compte. La tolérance du régime communiste vis-à-vis de l'Église orthodoxe se traduit par l'effort de faire bon ménage avec le haut clergé séculier (qui fut victime des persécutions de la première vague) et surtout par la présence, purement décorative, de la tête de l'Église aux côtés des chefs communistes, dans des situations bien sélectionnées (43).

Ces attitudes affichées tranchent avec le sort réservé au bas clergé, surtout dans la campagne. Jusqu'à la fin des années soixante, voire plus récemment encore, les popes des petites paroisses sont souvent victimes d'un traitement outrageant qui discrédite la "profession" (il est impossible de parler d'une vocation) de prêtre. Le clergé bulgare sort désorganisé et moralement déficitaire de la période trouble des persécutions. On est pourtant loin de la réalité des déportations massives de prêtres dans les camps dont font état les récentes révélations des archives du KGB. Après la répression initiale qui en conduit plusieurs à renoncer à la soutane, la réduction des offices au strict minimum et la fermeture de plusieurs églises villageoises (44) obligent ceux qui persistent à se partager entre plusieurs paroisses. Confiné à l'exercice d'un minimum de tâches sur des districts étendus où les vrais croyants se font de plus en plus rares, sans aucune marge de manœuvre, le prêtre de paroisse devient un marginal dans la vie sociale de l'époque. Toutefois, même lors des persécutions, les membres du clergé séculier restent des employés de l'État et continuent à percevoir des salaires fixes.

(41) Cf. Kalkandzhieva (1997, pp. 258-263). On retrouve cette stratégie chez d'autres Églises orthodoxes: pour une attitude analogue de l'Église orthodoxe en Roumanie, cf. GILLET (1997, pp. 109-118). (1989).

(42) Dès 1945, le Patriarche russe prêta main-forte pour la levée du «schisme bulgare» : cf. RAIKIN

(43) Ailleurs, on retrouve des logique semblables visant à attirer le soutien l'Église orthodoxe au profit des jeunes régimes communistes. Pour la Roumanie, c'est le procès contre l'Église uniate ou gréco-catholique et sa mise hors-la-loi - un «service» inestimable rendu par le pouvoir politique à l'Église orthodoxe (Gillet, 1997, loc. cit.). Pour les diverses manières d'instrumentaliser l'Église orthodoxe russe par Kremlin, cf. Meyendorff (1995, pp. 99-114); Rousselet (1997); Beauvisage (1998, pp. 30-35).

(44) Le plus souvent sans rapport avec les persécutions antireligieuses. Accompagnant la modernisation accélérée (et souvent forcée) de la campagne bulgare, l'industrialisation et l'exode rural font davantage dans ce sens que les interdits et les intimidations vis-à-vis des croyants. 


\section{Les rites : le religieux maîtrisé}

Sur le terrain, la "mort de la religion" annoncée par l'idéologie marxiste s'exprime dans des actions multiformes qui visent à priver l'Église du pouvoir exclusif de marquer les grands moments dans le cycle de vie. Cette tâche demande un dispositif subtil car, malgré les efforts d'élimination de la religion de la sphère publique et de la vie sociale, la ritualisation des grands tournants de la vie humaine répond à un besoin social (45). La solution consiste à soustraire ces derniers à l'Église et à les ré-instituer sous forme de rituels civils, tout en multipliant les entraves directes et indirectes à l'accomplissement du rituel d'Église respectif. Dans ce combat pour la gestion du sacré dans la vie humaine, la naissance et le mariage sont les cibles privilégiées. L'interdit pèse moins sur les rites funéraires et la commémoration des morts qui continuent à être très marqués par la pratique orthodoxe. Agissant dans un sens pratique qui peut être résumé par la formule "laisser aller ce qui s'en va», les funérailles constituèrent des occasions de force majeure pour l'ouverture de l'église et l'accomplissement d'un office sommaire (46). Cette relative liberté religieuse dont jouit le Bulgare dans le domaine de la mort et de la commémoration est à l'origine d'un glissement des activités rituelles observé sur le terrain. Funérailles et commémorations deviennent un pôle d'attraction pour les rites de passage traditionnellement liés aux autres "seuils" du cycle de la vie humaine : mariage, naissance, baptême (47).

L'effort de supplanter le religieux de la vie quotidienne s'exerce également sur la célébration des fêtes et des rites calendaires. Les fêtes sont "épurées" de leurs composantes chrétiennes censées être une "mince couche" recouvrant un "esprit païen" - "typiquement bulgare" et "intrinsèquement rebelle aux dogmes chrétiens" (48). Les restrictions les plus suivies sont appliquées aux fêtes christiques (surtout Noël et Pâques) et mariales, trop imprégnées de signification pour se prêter avec succès à une "conversion civile". L'interdit formel se double d'une surveillance sur les activités rituelles, particulièrement autour de Noël, du Carême-Prenant et durant la Semaine sainte. La symbiose entre fête religieuse et coutumes locales rend ces mesures particulièrement douloureuses. L'entrave de l'activité rituelle pendant la période de Noël est particulièrement mal vécue là où la fête donne lieu à des rassemblements familiaux, lesquels fonctionnent comme des repères majeurs de l'identité du groupe lignager. Les études effectuées à partir de 1990 montrent que, rétrospectivement, ce qui a le plus marqué les gens, était l'impossibilité d'exercer ouvertement les activités rituelles liées à la célébration de Noël et de Pâques, précisément en raison de leur caractère traditionnel.

D'autres fêtes liées à la commémoration de saints sont entièrement récupérées par le système rituel socialiste. Il s'agit de saints que la tradition orale a consacrés

(45) Il s'agit de cette "bataille pour la suprématie rituelle” (KERTZER, 1980, p. 131) que l'on retrouve, également, dans une société catholique parfaitement "occidentale" où l'idéologie marxiste eut des positions très fortes dans les années 1960-1970 : cf. KERTZER (1980, pp. 131-168).

(46) Pour la formule (d'ailleurs dérivée du mot d'ordre de la religion qui «se meurt») et pour une documentation sociologique de ce phénomène cf. OcHAVKov, éd., (1970, pp. 257-279).

(47) Cf. Garnizov (1992); l'infléchissement de la religion orthodoxe vers la mémoire et la commémoration est observé, également, en Grèce : cf. Hirschon (1983), SEREMETAKis (1991), Hart (1992, pp. 17-26; 171-192).

(48) Pour les qualifications cf. Draganov (1968, passim); Ochavkov, éd., (1970, pp. 132-149). 
comme patrons d'activités économiques (l'élevage, la viticulture, l'apiculture), réputations entretenues par une tradition orthodoxe assez prolifique mais floue. C'est précisément cette 'tradition populaire' qu'on peut facilement présenter comme la célébration d'une activité économique (49). L'aspect fête populaire conjugué à la dimension économique est une ressource puissamment exploitée aussi bien par le régime que par les acteurs sociaux ordinaires. Il permet de préserver une institution ancienne et très populaire comme le sâbor, à la fois pèlerinage, foire et pardon breton, que l'on essaie toutefois de dissocier du nom du saint respectif.

Confrontant les divers aspects de la mainmise sur la fonction rituelle de la religion, on peut dire que sa fonction de ciment du groupe est profondément perturbée. D'une manière générale, les restrictions sur les cérémonies d'église entraînent la déchéance ou la suppression d'aspects essentiels de la fête religieuse, tels que la liturgie ou l'observation d'un régime alimentaire rituel. La fête chrétienne est progressivement réduite à un divertissement folklorique. Elle n'est pas pour autant détruite : permettant à la fois de célébrer la communauté (professionnelle, mais aussi du lieu) et de ressourcer l'identité (locale ou nationale), la fête et les rites constituent le cadre d'une vie publique auquel le revirement politique fera aussitôt recouvrer sa dimension religieuse initiale.

\section{Le patrimoine : le religieux dissimulé}

Le mariage entre christianisme orthodoxe et identité nationale a conditionné, dans le cas bulgare, une autre stratégie "douce" de maîtrise des marqueurs de la religiosité chrétienne. Il s'agit de la récupération des églises et des monastères orthodoxes au titre du patrimoine historique et culturel et de leur réemploi comme musées ou lieux touristiques. Les premiers pas dans ce sens d'une politique culturelle, au début des années soixante, se font remarquer par des excès dont le plus mémorable reste la fermeture à toute activité religieuse du monastère de Rila - haut lieu de l'orthodoxie et centre d'un pèlerinage national, transformé en musée national de l'art religieux. Entre 1961 et 1970, les moines sont 'délocalisés' dans un village en Bulgarie du Nord, à proximité d'un autre monastère orthodoxe célèbre, où ils sont contraints de "fournir du travail socialement utile" en exerçant des métiers d'art. Peu après leur départ lors de la réouverture au culte du monastère de Rila, la région de leur exil devient à son tour célèbre avec ses 'traditions populaires' ... en matière d'arts du bois, et la cible d'une politique du patrimoine qui déploie les grands moyens. C'est la fondation, toujours au début des années soixante, d'un Institut National pour les Monuments de la Culture qui offre le cadre légal et scientifique de cette reconversion des églises et des monastères. Le critère de base dans la sélection des lieux de culte orthodoxe à préserver est leur ancienneté. Une priorité absolue est accordée à ceux de l'époque médiévale (IX $-\mathrm{XIV}^{\mathrm{e}}$ siècles), par ailleurs extrêmement rares; viennent ensuite ceux construits à l'époque ottomane. La quasi-totalité des églises et des monastères construits au XIX ${ }^{\mathrm{e}}$ siècle jusqu'à la

(49) Ainsi la Saint-Georges (6 mai) est fêtée comme "Jour de l'éleveur", la Saint-Trifon (14 février) devient "Jour du viticulteur", la Saint-Elie - "Jour de l'apiculteur", etc. 
Libération (1878) jouit du statut de “monument de l'art et de l'architecture bulgare à l'époque du Réveil national". Ils obéissent à un autre critère décisif : le fonctionnement du bâtiment du culte comme lieu de mémoire, essentiellement mémoire de la résistance anti-ottomane (50).

La transformation de l'église en lieu de mémoire, d'une mémoire habilement orientée vers la célébration de la nation, est un aspect de cette récupération. L'autre est le mouvement progressif vers l'esthétisation de l'église qui devient un espace d'art, un lieu qu'on est censé visiter pour contempler la beauté de la peinture ou des formes architecturales (51). Cela donne lieu à une double manipulation du lieu de culte à des fins idéologiques, qui vise à préserver un capital symbolique déjà constitué en le logeant sous une autre enseigne (52). Ce statut de "monument historique" impose une ritualisation secondaire du lieu de culte chrétien, conforme à l'histoire et à la mémoire nationale. Ici comme ailleurs (53), elle se donne comme la façon politiquement agréée, de vivre le religieux. Évoluant entre le lieu d'art et l'espace de mémoire, cette manipulation idéologique du religieux semble s'inscrire dans une tendance plus générale propre à la société contemporaine : à la suite de D. Hervieu-Léger (1993), on peut le désigner comme une 'religion pour mémoire'.

En sa qualité de patrimoine historique l'église, espace mémorial et artistique, nécessite un entretien permanent et connaît de nouvelles formes de fréquentation. Parmi celles-ci, on remarque la prééminence des visites organisées étroitement encadrées, surtout celles qu'effectuent les élèves des écoles; plusieurs font partie des itinéraires touristiques recommandés. Ainsi se multiplient les occasions d'y accéder en dehors de tout contexte cultuel, donc sans l'intermédiaire du clergé. Les observations ethnographiques montrent que les tâches d'entretien sont en général le fait des plus croyants. C'est souvent dans la même catégorie que se recrutent les «gardiens de la clé» de l'église paroissiale. Toujours disponibles, ces gardiens se sont peu à peu substitués aux prêtres. À la différence de ces derniers, habituellement absents de l'église (paroissiale ou monastique) en dehors des heures des rares offices ou des ouvertures réglementées, les gardien(ne)s se distinguaient par ce volontariat qui n'avait pas la notion du «temps de travail». Pour avoir accès à l'église, les gens pouvaient s'adresser à eux, y compris chez eux, à tout moment de la journée. Devant l'impossibilité de conjuguer tous les éléments d'une pratique correcte du culte, les croyants adaptaient leurs comportements et leur demande religieuse à ce qui était accessible. Pour beaucoup, l'accès à l'église hors service religieux était suffisant. Sous l'apparence de la visite d'un lieu de mémoire, il permettait d'accomplir les gestes de dévotion les plus élémentaires.

(50) Le terme de lieu de mémoire est pris dans la définition de NoRA (1984). La formule de "préserver la foi orthodoxe face à l'Islam" ottoman - thème commun à l'historiographie et aux sciences sociales en Bulgarie jusqu'à nos jours - se prête sans difficulté à une vision de la religion, et de l'institution de l'Église, comme résistance.

(51) Cf. pour cet aspect CUISENIER (1994).

(52) Comme le montre Chastel (1986), cette ambiguïté et la sémantique plurielle qui en découle font partie intégrante de la notion de patrimoine en France.

(53) À des degrés différents, cette tendance est repérable dans les autres sociétés orthodoxes : cf. Rousselet (1997) et Beauvisage (1998) (pour la Russie), ainsi que Hervieu-Léger (1993) dans une perspective plus vaste. 


\section{Les spécialistes religieux alternatifs ? "Vanga, voyante d'État" [communiste] (54)}

L'histoire et la carrière extraordinaire de la voyante de Petrié, alias «la Pythie bulgare », est peut-être la preuve la plus éclatante des arrangements opérés, derrière la façade de "l'athéisme scientifique», entre religieux et politique dans un pays communiste de tradition orthodoxe. Elle montre comment ce qui fut banni des années soixante comme «superstition » par le chœur du rationalisme progressiste et de l'idéologie marxiste (55), est récupéré dans les années quatre-vingt comme un avatar de "la religiosité pré-chrétienne» et célébré, dans un régime à prétention scientifique, comme «la connaissance de l'inaccessible» (56).

Baba [«grand-mère»] Vanga (Evangelia Dimitrova, 1911-1996) est «voyante» de 1941 jusqu'à sa mort. Pendant plus de 50 ans, elle voit le passé, prédit le futur, et parle avec les morts et les disparus. La demande de ses 'dons' est si forte qu'elle continue à accueillir les foules en dépit des persécutions dont elle fut, semble-t-il, victime dans la décennie 1948-1958. Dès le début des années soixante, le flux de visiteurs pose la question de la gestion de ses activités, laquelle trouve sa réponse dans son passage au service de l'État en 1967. Elle est intégrée au système à double titre : en tant qu'employée de la municipalité de Petricet et en tant que «chercheur» à l'Institut de "Suggestologie» (sic) de Sofia, créé pour légitimer "scientifiquement" son intégration dans les structures de l'État. Vers la fin des années soixante-dix, l'usage 'secret' de ses 'dons oraculaires' au plus haut niveau de la hiérarchie du Parti est un secret de Polichinelle. L'attention publique fixée durablement sur la voyante nécessite une nouvelle gestion de son image. Elle sera le fruit d'un discours élaboré par quelques éminences de l'intelligentsia du régime Zhivkov, qui ont recours à l'histoire et à la manipulation d'un vocabulaire religieux et «humanitaire» à la fois. Ce discours s'infiltre progressivement dans la couverture médiatique du "phénomène Vanga». À la fin des années quatre-vingt, lorsqu'elle est ouvertement sollicitée dans les affaires publiques, ses oracles sont régulièrement diffusés par les medias. C'est à ce moment que Vanga est consacrée comme 'la Pythie bulgare' (57). Cette référence à une figure religieuse de l'Antiquité grecque est fort ambiguë : le prestige de la Pythie de Delphes ne vient-il pas de l'usage politique que la cité fit de son art divinatoire? Il est difficile de faire mieux que cette comparaison implicite, si riche de sens, pour suggérer la portée pratique d'une religiosité réinventée par le socialisme bulgare.

La gloire de Vanga atteint son véritable apogée à l'époque postcommuniste. Alors que sa maladie prolongée ne lui permet plus de poursuivre ses activités de 'voyante', elle reste recherchée en sa qualité de gourou, d'amie politique, de conseillère. Elle devient une référence légitimatrice pour un éventail très large d'acteurs sociaux. L'étude 'scientifique' pratiquée sur les dons de Vanga dès les

(54) J'emprunte ici l'intitulé d'un article de Noël FAVRELIÈRE (communication personnelle) publié au début des années soixante-dix.

(55) Cf. Draganov (1968, passim); Ochavkov, éd., (1970, pp. 247-273).

(56) Une des premières études sur ce cas - celle d'un sociologue bulgare (DoBRIYANOv, 1993) se présente comme une série de preuves scientifiques de l'authenticité des 'dons extraordinaires' de la voyante.

(57) Pour les détails, cf. Valtchinova (1998). 
années soixante et leur reconnaissance d'utilité publique dans les années quatre-vingt font d'elle l'objet d'appropriations auto-légitimantes par de «nouveaux » guérisseurs et pratiquants de médecines alternatives. Assoiffée de reconnaissance sociale, en 1992 la Société des extra-sensoriels proclame Vanga 1'"Extra-sensoriel numéro Un (58)". Des hommes politiques de tous bords se pressent chez elle (ceux de la droite démocratique sont toutefois minoritaires) ou la consultent par personne interposée. Quelques-unes des figures les plus populaires $\mathrm{du}$ changement démocratique s'affichent comme ses amis de longue date. Les membres de l'ancienne nomenklatura ne l'oublient pas pour autant: certains de ceux qui l'ont fréquentée grâce à leur proximité avec les sommités du Parti communiste construisent cette expérience comme une "opposition secrète au régime».

L'orchestration savante de l'image de la voyante, de même que la tendance des intellectuels (et par la suite des hommes politiques) à se déclarer en sa faveur ou comme ses 'amis', s'inscrit dans un débat beaucoup plus vaste qui traverse la société entière. C'est le débat autour de la 'nature' de sa voyance et des images dont Vanga opère lors de ses séances. Mais il porte aussi sur 'les forces motrices' ou 'l'inspiration' de son activité de voyante. Dans ce débat, le clergé orthodoxe prend invariablement parti contre la voyante, condamnant ses activités comme inspirées par Satan. Cette position lui vaut la participation à des configurations sociales inédites. À l'époque socialiste l'EOB se retrouve ainsi dans le même chœur que les athéistes endurcis et la petite nomenklatura du Parti, soucieuse du respect des anciennes consignes de 'lutte contre la superstition' : tous accusent Vanga de charlatanisme. Après 1989 l’Église se trouve dans une situation encore plus difficile lorsque la voyante annonce son projet de construire une église. Le refus des popes de consacrer l'église de Vanga soulève l'indignation publique et se trouve stigmatisé par des intellectuels très influents, surtout lorsqu'un petit milieu de fondamentalistes orthodoxes (les vieux-calendaristes) se rallient à l'EOB. Finalement, ce sont les considérations pragmatiques, le taux de fréquentation extrêmement élevé de cette église, qui ont raison des scrupules de nature dogmatique.

La querelle autour de «l'église de Vanga» montre de façon claire que la voyante agit, et était considérée agir, comme un spécialiste religieux alternatif. De par ses activités, quelles qu'en soient les motivations ou leur explication, Vanga se situe en marge de la religion officielle stricto sensu mais dans la droite ligne d'une religiosité populaire. D'ailleurs l'heure des interviews venue, elle déclare avoir été depuis toujours une "bonne chrétienne", sans avoir éprouvé le besoin d'exprimer sa foi avec ferveur. Là encore, il s'agit d'une religiosité dont la pratique est confinée aux actes dévotionnels de base : brûler des cierges, adorer des icônes, faire le signe de la croix, qu'on peut qualifier comme une religiosité quotidienne standard.

En conclusion, on se permettra de formuler une vision sur l'évolution de la vie religieuse en Bulgarie socialiste, soit grosso modo entre 1945 et 1990, qui dérange l'idée préconçue d'une rupture totale ou d'une fracture infranchissable par rapport à la période antérieure au régime communiste. Les aspects qu'on vient d'examiner montrent la difficulté à interpréter les développements de la vie religieuse unique-

(58) Cf. Dimov (1994, pp. 61-62). Une première réflexion sur les 'extrasensoriels' comme phénomène social est ébauchée dans le numéro spécial de la revue Bulgarski Folklor [Folklore Bulgare] 1995 , 5: "Le Paranormal". 
ment en termes de «mort» du religieux. En dépit des principes proclamés haut et fort par la doctrine marxiste en matière de religion, le soi-disant opium des peuples ne fut pas arraché à la racine. D'une façon générale, la répression ne s'exerce de façon dure que dans un nombre de cas limité, certes très choquants pour les mentalités occidentales, et essentiellement dans la période allant jusqu'au début des années soixante. Dans la vie quotidienne, aussi bien avant qu'après cette limite, elle peut s'exprimer de mille manières, agissant sur les figures formelles de la religiosité chrétienne. Il s'agit d'une série de restrictions qui, mis à part les cas flagrants, donne à la répression communiste en Bulgarie un aspect proche de la sécularisation dans laquelle les sociétés de l'Europe occidentale se sont engagées, pour la plupart après la Deuxième Guerre mondiale.

La relation entre politique et religieux à l'Est n'est réductible ni à un ensemble défini d'activités qui seraient l'expression d'une religiosité 'standard', ni au rapport Église-État : les deux peuvent varier largement en fonction des contextes culturels, historiques et politiques (59). D'un côté, l'encadrement idéologique du régime met en jeu certaines formes et concepts d'un anticléricalisme ancien qui, comme dans le contexte des sociétés catholiques, peuvent être manipulés à des fins (et par des idéologies) politiques. De l'autre, il préserve la coquille institutionnelle et une partie du contenu, à savoir les manifestations religieuses jugées utiles pour le maintien du "sentiment national". Les lieux sacrés de l'orthodoxie demeurent les lieux d'une mémoire valorisée par une idéologie qui reste nationaliste en dépit des slogans.

Usant tour à tour de la répression et des relectures périodiques de ses principes idéologiques, le régime communiste bulgare fait évoluer la vie religieuse vers des expressions plus feutrées et discrètes. Pour nous, la logique du vécu communiste du religieux consiste en une alternance, en fonction des contextes socio-économiques et politiques, entre différentes façons de maîtriser et de dissimuler le religieux, ou tout simplement de l'ignorer, en l'appelant autrement. Ce qui lui réserve une marge d'évolution non-négligeable au sein de la société bulgare à l'époque socialiste.

Galia VALTCHINOVA

Académie bulgare des sciences - Sofia

(59) C'est Patrick Michel $(1988,1994)$ qui souligne ce point en insistant sur les rôles différents joués par l'Église catholique en Pologne, en Hongrie et en Tchéchoslovaquie, à l'époque communiste. 


\section{BIBLIOGRAPHIE}

ARNAKIS George, " The Role of Religion in the Development of Balkan nationalism », in Charles and Barbara JELAVICH, eds. The Balkans in Transition. Essays on the Development of Balkan Life and Politics since the $18^{\text {th }}$ century, Berkeley (CA.) University of California Press, 1963, pp. 115-144.

BAZIN Louis, «La Turquie et la Grèce », in Jean DELUMEAU, dir., L'acceptation de l'autre. De l'Édit de Nantes à nos jours, Paris, Fayard, 2000, pp. 180-189 (coll. «Direction des Archives de France»).

BEAUVISAGE Laurence, La croix et la faucille. La religion à l'épreuve du postsoviétisme,Paris, Fayard, 1998.

BEYER Peter, «Privatization and the Public Influence of Religion in Global Society », Theory, Culture and Society, VII, 1990, pp. 373-395.

CAMPBELL John, Honour, Family and Patronage. A Study of Institutions and Moral Values in a Greek Mountain Community, Oxford, Clarendon Press, 1964.

CHASTEL André, "La notion de patrimoine», in Pierre NORA, dir., Les Lieux de Mémoire. ** La Nation, Paris, Gallimard, 1986, pp. 405-450.

CIPRIANI Roberto, ed., « Religions sans frontières? » Present and Future Trends of Migration, Culture, and Communication, Rome, Prezidenza del Consiglio dei ministri, 1994.

CRAMPTON Richard, A Concise History of Bulgaria, Cambridge, Cambridge University Press, 1997.

CUISENIER Jean, «Saints bulgares après le communisme : pour une autre iconologie », in L'art de la recherche. Essais en l'honneur de Raymonde Moulin, Paris, La Documentation Française, 1994, pp. 53-69.

CREED Gerald, Domesticating Revolution: From Socialist Reform to Ambivalent Transition in a Bulgarian Village, University Park, The Pennsilvania State University Press, 1998.

DAVIE Grace, La religion des Britanniques. De 1945 à nos jours, Genève, Labor et Fides, 1996.

DAVIE Grace, HERVIEU-LÉGER Danièle, dir., Identités religieuses en Europe, Paris, La Découverte, 1996.

DIMOV Vasil, « Devenir extra-sensoriel », Bulgarski folklor (Folklore bulgare), 1994, 6, pp. 57-65.

DOBRIJANOV Velicko, Phenomenat Vanga [Le phénomène Vanga], Sofia, Éditions de 1'Université St. Kliment Okhridski, 1993.

DRAGANOV Minco, Religioznata psihika na bâlgarite [Le psychisme religieux des Bulgares], Sofia, Partizdat, 1968.

DUBISCH Jill, "Gender, Kinship and Religion: 'Reconstructing' the Anthropology in Greece », in Peter LOIZOS, Eftimios PAPATAXIARCHIS, eds., Contested Identities, Gender and Kinship in Modern Greece, Princeton, Princeton University Press, 1991, pp. 29-47.

DUBISCH Jill, In a Different Place. Gender, Pilgrimage and Politics on a Greek Island, Princeton, Princeton University Press, 1995.

FILATOV Sergei, «On Paradoxes of the post-communist Russian Orthodox Church», in Roberto CIPRIANI, ed., Religions sans frontières?, 1994, pp. 117-125.

FOTEV Georgi, Krisis na legitimnostta [Crise de la légitimité], Sofia, Éditions de L’Université St. Kliment Okhridski, 1999.

GARNIZOV Vassil, «Identité et rites des funérailles à l'époque du totalitarisme», Études et documents balkaniques et méditerranéens, 10, 1992. pp. 68-70.

GILLET Olivier, Religion et nationalisme. L'idéologie de l'Église orthodoxe roumaine sous le régime communiste, Bruxelles, Éditions de l'Université de Bruxelles, 1997.

HART Laurie K., Time, Religion, and Social Experience in Rural Greece, New York, Rowman and Littlefield Publishers, 1992.

HERVIEU-LÉGER Danièle, La Religion pour Mémoire. Paris, Cerf, 1993.

HERZFELD Michael, Cultural Intimacy. Social Poetics in the Nation-State. New York-Londres, Routledge, 1997[a].

HERZFELD Michael, «Segmentation and Politics in the European Nation-State. Making Sense of Political Events », in Kirsten HASTRUP, ed., Other Histories. Oxford-New York, Routledge, 1997 [1997b], pp. 62-81. 
HIRSCHON Renée, «Women, the Aged and Religious Activity: Oppositions and Complementarity in an Urban Locality », Journal of Modern Greek Studies, I, nº 1, May 1983, pp. 113-129.

JUST Roger, «Anticlericalism and National Identity: Attitudes Towards the Orthodox Church in Greece ", in Wendy JAMES, H. Johnson DOUGLAS, eds., Vernacular Christianity. Essays in the Social Anthropology of Religion, Oxford, 1988, pp. 15-30.

KALKANDZHIEVA D., Bälgarskata pravolsavna cârkva i dârzhavata, 1944-1953 (L'Église orthodoxe bulgare et 1'État, 1944-1953, Sofia, Albatros, 1997.

KERTZER David, Comrades and Christians: Religion and Political Struggle in Communist Italy, New York, Cambridge University Press, 1980.

KOKOSALAKIS Nikos, "The Political Significance of Popular Religion in Greece», Archives de Sciences Sociales des Religions, 64 (1), 1987a, pp. 37-52.

KOKOSALAKIS Nikos, "Modernization in $19^{\text {th }}$ century Greece», Social Compass $34, n^{\circ} 2-3,1987 \mathrm{~b}$, pp. 223-241.

KOKOSALAKIS Nikos, "Orthodoxie grecque, modernité et politique», in Grace DAVIE, Danièle HERVIEU-LÉGER, dir., Identités religieuses en Europe, Paris, La Découverte, 1996, pp. 131-151.

LORY Bernard, L'Europe balkanique de 1945 à nos jours, Paris, Ellipses, 1996.

LUCKMANN Thomas, The Invisible Religion, New York, MacMillan, 1967.

LÜSCHEN Gerhard, STAJKOF Zahari, STOLTE HEISKANEN Veronica, WORD G. «Family, Ritual and Secularization. A Cross-National Study Conducted in Bulgaria, Finlandia, Germany and Ireland», Social Compass, XIX, 4, 1972, pp. 519-536.

MAKRIDES Vasilios N., «Orthodoxy as a Conditio sine qua non: Religion and State/Politics in Modern Greece from a Socio-Historical Perspective», Ostkirchliche Studien, Bd. 40, Heft 4, 1991, pp. 281-305.

MEYENDORFF Jean, L'Église orthodoxe hier et aujourd'hui (Nouvelle éditon revue et augmentée par J. MeyendorfF et Nicolas Lossky), Paris, Seuil, 1995.

MICHEL Patrick, La société retrouvée. Politique et religion dans l'Europe soviétisée, Paris, Fayard, 1988.

MICHEL Patrick, Politique et religion. La grande mutation, Paris, Albin Michel, 1994.

NORA Pierre, «Entre Mémoire et Histoire, la problématique des lieux », in Les Lieux de Mémoire. I. La République, Paris, Gallimard, 1984, pp. XVII-XLII.

OCHAVKOV Zhivko [sous la réd.], Procesât na preodoljavaneto na religijata v Bâlgarija. Sociologicesko izsledvane (Le processus d'évanouissement de la religion en Bulgarie. Étude sociologique), Sofia, Éditions de l'Académie Bulgare des Sciences, 1970.

PAPASTHATIS Charalambos, «La République hellénique», in Brigitte BASDEVANT-GAUDEMET, Francis MESSNER, dirs., Les origines du statut des confessions religieuses dans les pays de l'union européenne, Paris, PUF, 1999, pp. 207-221.

PETROV Petar, «Conquering the Feast. The Socialist Transformation of a Religious Feast in a Bulgarian Village », Ethnologia Balkanica 2, 1998, pp. 127-136.

RAIKIN Spas, "Nationalism and the Bulgarian Orthodox Church», in Sabrina P. RAMET, ed., Religion and Nationalism in Soviet and East European Politics, Duke, Duke Press Policy Studies, 1989, pp. 352-377.

RAMET Sabrina P. Balkan Babel. Politics, Culture and Religion in Yugoslavia, Boulder (CO), Westview Press, 2e édition [1 ère éd. 1992], 1996

RÉMOND René, «Anticlericalism: Some Reflections by Way of Introduction», The European Studies Review, vol. 13, no 2, avril 1983, nº spécial 'Anticlericalism', pp. 121-126.

ROUSSELET Kathy, «L'Orthodoxie et le déficit démocratique russe », in Patrick MICHEL, dir., Religion et démocratie. Nouveaux enjeux, nouvelles approches, Paris, Albin Michel, 1997, pp. 257-274.

SELLS Michael A., The Bridge Betrayed. Religion and Genocide in Bosnia, Berkeley (CA), University of California Press, 1996.

SEREMETAKIS C. Nadia, The Last Word. Women, Death and Divination in Inner Mani, ChicagoLondres, The University of Chicago Press, 1991.

STEWART Charles, "Who owns the Rotonda? Church vs. State in Greece », Anthropology Today, 14, $\mathrm{n}^{\circ}$ 5, 1998, pp. 3-9. 
SUGAR Peter, «The Historical Role of Religious Institutions in Eastern Europe and Their Place in the Communist Party-State», in Sabrina P. RAMET, ed., Religion and Nationalism in Soviet and East European Politics, Duke, Duke Press Policy Studies/Duke University Press, 1989, pp. 42-58.

TODOROVA Maria, Imagining the Balkans, New York-Oxford, Oxford University Press, 1997.

TOMKA Miklós, «Secularization or Anomy? Interpreting Religious Change in Communist Societies », Social Compass, vol. 31, 1, 1991, pp. 93-102.

VALTCHINOVA Galia, «Entre religion, politique et tourisme : le pèlerinage de Rupité, en Bulgarie », Ethnologie Française 3, 1998, pp. 396-405.

VARGA Ivan, «Orthodoxy, Modernity and Post-Communism», in Roberto CIPRIANI, dir., Religions sans frontières?, Rome, Prezidenza del Consiglio dei ministri, 1994, pp. 144-149.

VRCAN Srdjan, «A Christian Confession seized by nationalistic paroxysm: the case of Serbian Orthodoxy » in Roberto CIPRIANI, dir., Religions sans frontières ?, Rome, Prezidenza del Consiglio dei ministri, 1994, pp. 150-166.

WHITE Stephen, Ian McALISTER, « The Politics of Religion in Postcommunist Russia », Religion, State \& Society, vol. 25, no 3, 1997, pp. 235-252. 


\section{Résumé}

L'article propose un regard critique sur la thèse du vide religieux qui aurait prévalu dans les sociétés communistes, laquelle sous-tend le concept de renouveau religieux que ces sociétés vivent actuellement. Il se propose d'en tester le bien-fondé à partir de l'exemple de la Bulgarie, un pays balkanique à dominante orthodoxe où le conflit religieux demeure latent, et qui est reconnu comme l'État le plus réceptif à l'influence soviétique.

L'argumentation cherche à faire ressortir les divers aspects de la relation entre religieux et politique à l'époque communiste : le lien entre modernité, anticléricalisme et sécularisation; les implications religieuses de la cö̈ncidence entre la modernisation d'une société et son développement sous un régime politique totalitaire; la marge d'évolution du religieux dans une société athée par définition où la confession continue à fonctionner comme repère identitaire. En conclusion, on propose de voir le vécu religieux comme l'alternance de différentes stratégies politiques : maîtriser, dissimuler ou ignorer la religiosité chrétienne, ce qui lui réserve une marge d'évolution non-négligeable au sein de la société bulgare à l'époque communiste.

\section{Abstract}

The paper examines critically the vision about the religious vacuum in the communist societies, thereby calling for a more nuanced appraisal of the post-totalitarian religious revival. With its characteristics of a Balkan and dominantly Christian-Orthodox society with latent religious conflict - on the one hand, and traditionally perceived as the most docile Soviet satellite - on the other, Bulgaria appears to be particularly appropriate for testing this vision.

We try to bring together and to make intersect differents aspects of the relationship between religion and politics under socialism: the conflation of modernity, anticlericalism and secularisation; the religious implications of the modernising process under a totalitarian regime; the margins of evolution of religious life in a society that exhibits an "atheistic" ideology but in which religion continues to play a central role in the construction of national identity. After discussing these issues, we propose a more complex vision of religious life under socialism in which trends towards repression alternate with various subtle strategies for dominating the course of religious life and remodelling its expressions, thus contributing to its further evolution.

\section{Resumen}

El artículo propone una mirada crítica sobre la tesis del vacio religioso que habría prevalecido en las sociedades comunistas, que subyace al concepto de renovación religiosa que estas sociedades viven actualmente. Trabajaremos la hipótesis a partir del ejemplo de Bulgaria, un país balcánico predominantemente ortodoxo, en el que el conflicto religioso permanece latente, y que es reconocido como el Estado más receptivo a la influencia soviética.

La argumentación trata de hacer surgir los distintos aspectos de la relación entre lo religioso y lo político en la época comunista: el lazo entre modernidad, anticlericalismo y secularización, las implicancias religiosas de la coincidencia entre modernización de una sociedad y su desarrollo bajo un régimen político totalitario, el margen de evolución de lo religioso en una sociedad atea por definición, donde la confesión religiosa sigue funcionando como marcador identitario. En conclusión, nos proponemos ver las vivencias religiosas como la alternancia de diferentes estrategias políticas : dominar, disimular o ignorar la religiosidad cristiana, lo cual le reserva un margen de evolución nada despreciable en el seno de la sociedad búlgara en la época comunista. 
